\title{
Successful Repair of Ruptured Abdominal Aortic Aneurysm: A Case Report
}

\author{
Dr. Jyotindra Sharma, Dr.Ramesh Raj Koirala, Dr.Anju Shrestha, \\ Dr.Sidhartha Pradhan, Dr.Bhagawan Koirala
}

\section{Introduction:}

Abdominal aortic aneurysms (AAA) are the commonest arterial aneurysms being located mostly in the infrarenal aorta (1). Though pathogenesis of AAA is multifactorial including genetic predisposition, aging, atherosclerosis, inflammation and localised proteolytic enzyme activation, atherosclerotic degeneration of the arterial wall is the most common cause for this. Though other complications like thromboembolism may occur, AAA gains its importance mostly because of the fear of rupture. Aneurysm rupture is the cause of death in $1.2 \%$ of males and $0.6 \%$ of females in the United States. It is the $13 t$ " most common cause of death in United States killing about 15,000 people annually. Once ruptured the condition carries a high degree of mortality even in the busy centers.

\section{Case Summary:}

A 54 years old gentleman presented from Gulmi to our emergency on 2058/ 11/15 BS (Feb.27, 2002) with complain of continuos pain abdomen for 3 days associated with one episode of vomiting. He also gave $\mathrm{H} / 0$ distension of abdomen for 1 day, decreased urinary output for 12 hours. He was taken to Palpa Tansen hospital 2 days after the onset of pain where he was rescucitated and managed with I/V fluids/antibioties. USG abdomen done showed AAA and so they referred the patient to Kathmandu. But unfortunately he had to be taken from one hospital to another and he finally landed at our hospital 12 hours after he arrived in Kathmandu. On examination, the patient was in profound shock, was delirious with GCS of $11 / 15$. He was markedly pale, pulse and R/R were 120 and 28 per minute respectively, B.P at right arm, left arm and right leg were $100 / 80,90 / 70$,and $140 / 100 \mathrm{mmHg}$ respectively, He had scanty red urine in the urobag suggestive of haematuria.

On per abdominal examination, the abdomen was tender, there was a pulsatile abdominal mass In the central abdomen approximately $8 \times 10 \mathrm{~cm}$ in size, bowel sound was present but sluggish and there was a systolic bruit heard in the epigastric region. On auscultation of precordium, both heart sounds were audible with no murmur. All the peripheral pulses were palpable except the left dorsalis pedis. His haemoglobin 


\section{- NEPALESE HEART JOURNAL •}

was $7.2 \mathrm{gm} \%$, serum urea $18.0 \mathrm{mmol} / \mathrm{l}$, serum creatinine $241 \mathrm{micro} \mathrm{ml}$, Blood sugar and electrolytes were within normal limit With all these history and findings we had a diagnosis of contained rupture of AAA with renal involvement and ARF, and we decided to operate on him. With the minimum investigations of haematology and biochemistry at our hospital, we rushed him to operation theatre.

Abdomen was opened with upper and mid-line incision and the aorta was approached. There was about $300 \mathrm{ml}$ of haemorrhagic fluid in the peritoneal cavity There was a huge retroperitoneal haematoma around the abdominal aorta with a huge AAA, extending ju from the infrarenal aorta to the bifurcation, Proximal aorty was taken into control at the lavel of diaphragma Distally both femoral arteries were taken into contral With both proximal and distal control with aortic cro clamp, the aneurysm was opened and all the blood and clot sucked out. Aorta was badly diseased wil atherosclerotic plaques and was very fragile, There was a tear of $2 \mathrm{~cm}$ in the proximal aneurysmal part. A $8 \mathrm{~cm}$ long, $16 \mathrm{~mm}$ diameter PTFE tube graft was used to reconstruct the aorta from the nack of the aneurysma sac superiorly to just proximal to bifurcation distally with continuos $2 / 0$ prolene sutures in 30 minutes of aorti cross clamp. The aneurysmal sac was sutured over the graft. The abdomen was closed without a drain. Throughout the operation, the patient's blood pressur was maintained at a lower side with the help of sodium nitroprusside, nitroglycerine infusion drip and vasopressores.

\section{Postop period:}

The patient was severely acidotic during and just after the operation with a $\mathrm{PH}$ of 7.2 and base excess of $-13.2 \mathrm{mmol} / \mathrm{l}$. The patient was treated with soda bicarbonate, was paralysed and ventilated overnight to correct his acidosis. The patient was' extubated 17 hours after the operation.

The patient's blood pressure was maintained between 140-1 50/80-90 mm Hg in immediate postoperative period with the help of Sodium Nitroprusside, GTN drip followed by oral captopril and metoprolol from $2^{\circ} \mathrm{d}$ postoperative day. He remained delirious for 48 hours after extubation.

The patient was given IV lasix during and after operation to induce urinary output. On the day of operation, he received $80 \mathrm{mg}$ IV lasix, $60(20+40) \mathrm{mg}$ on $1^{\text {st }}$ POD after which he entered into diuretic phase of ARF producing $5742 \mathrm{mi}$ urine on $2^{\text {nd }}$ POD. His urea and creatinine were $20 \mathrm{mmol} / \mathrm{L}$. and 325 micromot/L respectively on $1^{\text {st }} \mathrm{POD}$ which gradually decreased to $8 \mathrm{mmol} / \mathrm{L}$ of urea and $127 \mathrm{rhicromol} / \mathrm{L}$ of creatinine on discharge. 
Patient started passing bloody mucoid diarrhoeal stool from 2" POD most likely due to ischaemic colitis. It was managed with ORS and IV fluids with addition of metronidazole, with spontaneous resolution over 3-4 days, The patient was fully oriented, mobile and was taking orally when he was discharged on $10^{\text {th }}$ POD. F/U: On follow up after 1 month and 6 months of operation, his B.P. was 120/80 $\mathrm{mmHg}$ and all the pulses were palpable except left dorsalis pedis. His wound was good and there was no lump or bruit per abdomen.

\section{Discussion:}

The most dreaded complication of AAA is the rupture and ironically the natural history of AAA Is to enlarge and rupture. The single most important factor associated with rupture is maximal cross-sectional aneurysm diameter. Aneurysms enlarge at an average rate of $0.4 \mathrm{~cm}$ per year with a higher individual variability (2). The risk of rupture is estimated at $1-3 \%$ per year tor aneurysms 4 to $5 \mathrm{~cm}, 6-11 \%$ per year for 5 to $7 \mathrm{~cm}$ aneurysms and $20 \%$ per year for aneurysms greater than $7 \mathrm{~cm}$ (2). Aneurysms smaller than $4 \mathrm{~cm}$ appear to be at a very low risk of rupture. Arterial hypertension and COPD increase the risk of rupture. Here in our patient, we do not know the size of the aneurysm before rupture though at operation the size was $8 \mathrm{~cm}$ and moreover he was a known hypertensive for quite a few years for which he was not taking medications. He was a smoker and had COPD also. Rupture of AAA can be either anterior or posterior. About $20 \%$ of AAA rupture are anterior (free intraperitoneal rupture) which causes immediate hemodynamic collapse and has a very high mortality rate $(>90 \%)$. About $80 \%$ of AAA rupture are posterior (retroperitoneal) and are contained by psoas muscle and adjacent periaortic and perivertebral tissue because of which they may be hemodynamically stable at the time of prasentation, and so they have a mortality figure of around $50 \%$ (3). Our patient was lucky to have a posterior retroperitoneal rupture and to survive for more than 48 hours before he presented to us. Though he was in ARF and was disoriented, his blood pressure was relatively maintained when he presented to us.

The only treatment of ruptured AAA is immediate surgical repair. If the patient if in shock and has an expansile abdominal lump suggestive of ruptured AAA, the patient can be rushed to the operation theatre without doing any imaging investigations. The preferred approach is transperitoneal with either a midline or a transverse abdominal incision. We operated on this patient via transperitoneal approach by a midline abdominal incision. A' $16 \mathrm{~mm}$ Dacron graft was used for the repair.

The survival statistics after rupture of an aortic aneurysm have not changed significantly since 1980s. Following rupture of an AAA, only $50 \%$ of patients arrive at the hospital 
alive. Of these, $24 \%$ of more die before surgery and $42 \%$ die after the operation, for an overall mortality rate of 78 to $94 \%$, But mortality is $100 \%$ if not operated $(4,5)$. The mode of death is usually due to multi organ dysfunction (MOD) (4). Our patient was a very high risk case due to very late presentation, acute renal failure with haematuria, disorientation and moreover he was a hypertensive as well as a COPD patient. On the one hand, with all this background there is always a hesitancy to operate on these patients while on the other he would have surely died if not operated.

In conclusion, it seems possible to salvage these difficult patients even in our setup, however early referral to an appropriate centre is necessary.

\section{Reference:}

1. Johnston KW, Rutherford RB, Tilson MD, et al: Standards for reporting on arterial aneurysms, J Vase surg 13: 452-458,1991.

2. Bernstein EF, Dilley RB, Goldberger LE, et al: Growth rates of small abdominal aortic aneurysms, surgery 80:765-773, 1976.

3. Lorenzi M, Coratti A, Tani F; Succesful surgical repair of a ruptured abdominal aortic aneurysm in a nonagenarian. IntAngiol 2001 Dee; 20 (4): 351-3.

4. Darling RC 3,d, Cordero JA Jr, Chang BB, et al: Advances in surgical repair of ruptured abdominal aortic aneurysm. Cardiovasc surg 1996 Dee! 4(6); 720-3,

5. Mulherin JL Jr, Edwards WH: Improved survival after ruptured abdominal aortic aneurysm. South Med J 1980 Aug; 73 (8): 986-9, 